\title{
SIMARECH 3: A New Application for the Governance of Scientific Research
}

\author{
Khaoula Benmoussa ${ }^{1}$, Majida Laaziri ${ }^{1}$, Samira Khoulji ${ }^{1}$ and Kerkeb Mohamed Larbi ${ }^{2}$ \\ ${ }^{1,2}$ Information System Engineering Resarch Group \\ ${ }^{1}$ National School of Applied Sciences of Tetouan. \\ ${ }^{2}$ Faculty of Sciences of Tetouan. \\ Abdelmalek Essaâdi University \\ Benmoussakhaoula24@gmail.com, majida.laaziri@gmail.com,khouljisamira@gmail.com, \\ kerkebml@gmail.com
}

\begin{abstract}
In the international context of research assessment, many countries appear to move critically towards a quantitative evaluation policy, which would eventually complement or replace the traditional peer review system. However, infometrics studies, based on the running of large international scientific databases, are confused by the complex organization of their research system. Furthermore, these databases prove to be inadequate to study the local research institutions and units. After a description of the current situation and a needed analysis survey, this proposal presents the design and development of an information system conceived as a tool for monitoring the research carried out by a university or another institution. Based on the use of an effective application developed by a team of Abdelmalek Essaâdi University(UAE) dedicated to conduct collaborative extranet, this information system, called SIMArech "Système d'Information de la Recherche Scientifique", is fully customizable in "real-time". It is available in Arabic, English and French and its flexibility enables it to dynamically adjust to the specificities of any institution having research units. Actually, several universities in three countries are implementing this system to improve their management of scientific research. Several examples are given to demonstrate how this tool is able to continuously develop a "state of the art" or a "dashboard" for these structures and their scientific production. The emphasis is put on the fact that local research structures, as well as national structures, needless research evaluation than valorization of their research.
\end{abstract}

\section{Introduction}

Higher education has long been a strongly competitive matter. Governments and university alike keenly watch the rankings produced many multitudes of organism; there aremore than 150 national rankings around the world. Such rankings encourage stratification. One of the metrics is the scientific production that accentuates the differentiation between better and worse universities. The North African model is thus quite different from the European or the North American one, which is a lot less selective and more homogeneous [1]. 
Because of a high globalizationof the scientific research and the growth in international cooperation[2], attention has shifted from national to international rankings.Governments want top-class universities because the modern economy is driven by scientific research and high-qualified human capital.

Both public and private universities that seek research funding from government and foundations must provide evidence of their accomplishments and capacities. These universities must account for their performance as part of professional accountability protocols[3]. Indeed, every university must have a clear and an evidence-based understanding of the institution's performance towards its goals and mission.

This understanding is achieved and maintained through an ongoing evaluation of all the institution functions. Meanwhile, because research is a central function, the university must evaluate its performance. Data on research performance helps to inform strategic decisions about what areas of research to support or build. It also helps the university leaders understand the institution's position relative to global and domestic standards of research production[4].

Every university must know how much research is conducted, and what its impact was, how many articles of university members were published and in which class of journals, etc. In fact, there are many international databases and editors dedicated to counting, measuring, and comparingthe scientific articles produced by members of universities[5]. However, the research results through publications has become enormous and complex, and itshould not be restricted to scientific articles but should include also several other categories such as thesis, communications, books, prizes, research projects, motilities, patents, facilities, equipment and services etc.In fact, it is so complex and specialized that personal knowledge and experience are no longer sufficient tools for understanding trends or for making decisions by universities or governments[6].

Government and universities must be able to make good decision about what research should be supported and what should not, or which research projects and researchers should receive more support than others. For a good piloting of the scientific research, including all its components, an information system becomes an obligatory requisite to manage better investments in scienceto evaluate the research performance and to establish a sound policy for the development of scientific research[7].

\section{The information system SIMArech}

Based on the use of an effective application developed by a team of the UAE dedicated to conduct collaborative extranet, this information system, called SIMArech, aims to support research administration by a unique technology solution in order to allow a streamlined and single-point access to up-to-date information for researchers, research staff and administrative staff.

SIMArech is indeed the result of a collaborative way of working. This innovative best-of-breed solution has been designed to fulfill the needs of academic andresearch institutions: SIMArechis an IT platform that makes it easy to collect and manage data on research activitiesand outputs within an organization. Researchers, administrators and evaluators are given all thetools needed to monitor research results, enhance visibility and efficiently allocate available resources.

The modular nature of the system and the flexibilityof its data model facilitate processing, organizing and transmitting information in accordancewith international standards. At the same time,SIMArech provides the chanceto easily shape local and national requirements. 
The compliance with international standards and databases of the Web of Scienceoffers several advantages toSIMArech: it strengthens relations amongresearch entities (people, resources, activities,publications, etc.); it supports evaluation activitiesand dissemination; it helps the exchange ofinformation among different Research Systems at national and internationallevels.

Modules are natively integrated with the IT infrastructureof the organization, both with legacysystems (such as HR, accounting databases) andexternal systems (regional, national and internationaldatabases) so that Return on Investment- ROI- is always maximized.

SIMArech supports research by delivering and maintaining innovative and integrated systems that simplify administrative processes in order to:

- Reduce administrative burden.

- Streamline and automate research administration.

- Provide authorized individuals and systems with convenient access to timely information regarding proposals, awards, and compliance.

- Improve the efficiency of administrative offices to meet the demands of a growing workload with limited resources.

- Make the pilotage process of the research addressed faster, easier to understand, and to improve the efficiency of surrounding processes.

- Making the process more transparent by reporting on research status, information sharing and easy data integration between university management systems.

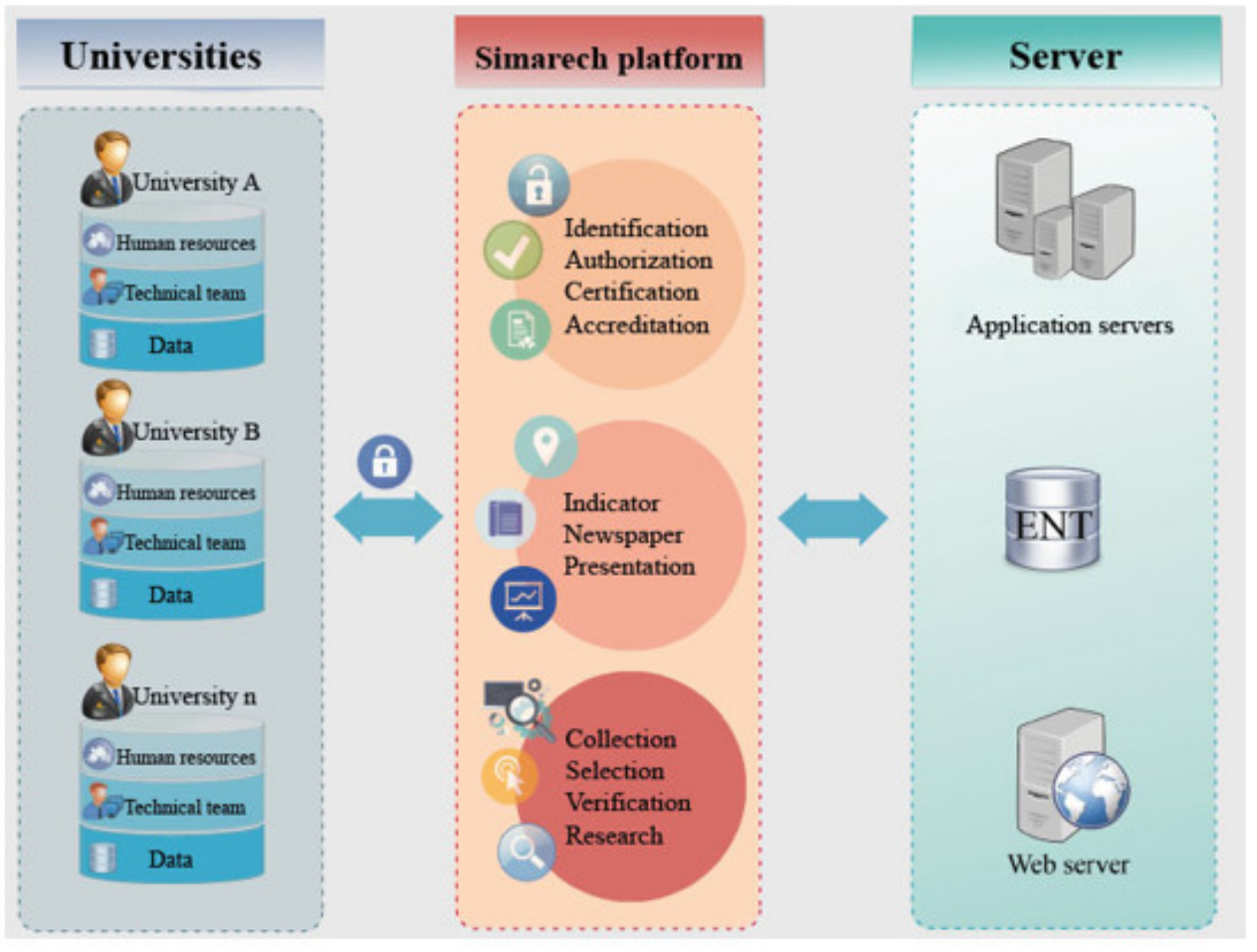

Figure 1: Integration of SIMArech in universities 
SIMArech was designed to meet the specific needs of African universities and Governments by:

- Promoting and increasing the visibility, exchanges and communication between scientists and researchers at regional, national or international level.

- Organizing and maintaining unified science and technology registries to produce accurate, reliable and up-to-date statistics in real time.

- Assisting in the overall management of researchers in the research units of the institutions and their scientific results.

- Presenting coherently the research units and their environment.

- Providing up-to-date information on individual research programs.

- Exchanging knowledge at the national and international level, in order to enrich the cultural and professional experience of researchers

- Ensuring interoperability between national scientific and technological databases.

- Establishing a knowledge base that is authorized to submit standardized criteria, and is agreed upon in the evaluation and quality of the results of the scientific activity.

\section{The SIMArech Technologies:}

SIMArech is based on open-source technologies that the UAE team contributes to develop and maintain. Itwas developed using a Framework called Symfony which was quickly adopted by professionals active in this field following its launch in 2005. This Framework today is a stable environment that is both wellknown and recognized internationally. Basically, Symfony is a framework chosen by the community; thus, it can take advantage of all the efforts of the OS developers around it: from automated deployment tools to fully integrated ORMs, from testing frameworks to tutorials and best practices, through native, advanced debugging tools. Symfony is as of today the most complete framework available in the PHP ecosystem when you take in consideration learning curve, integrations, stability and performances. The number of its references attests to this as they have grown significantly since its launch. This Framework is also an active community; developers, integrators, users and other contributors participate in the ongoing enrichment of this tool[9-10].

The choice of Symfony as a framework was based on the fact that it is well decoupled, so unit testing becomes very easy since it is possible to mock objects, isolate classes and inject stub dependencies very easily. It provides a first layer for functional testing (with PHPUnit): being an HTTP-centric framework. It provides also a base class that lets simulating HTTP requests and examines the output.

At the end, we can see how Symfony and the ecosystem around it provide the proper toolset to run unit, functional and behavioral tests. From the beginning, we take care of testing and we do not want to waste a huge portion of time doing manual testing. For this reason, we looked for it to take advantage of technology to automate heavy tasks that harms the application development.

This Framework has also a very powerful debug toolbar that would present all these information in order to ease debugging. Database inspection permit to realize how many queries are running and see the SQL of all of them with a nice overview of the time they take, while the profiler itself includes information about every step of the application. Since we are working with a Service-Oriented Architecture, we take advantage of easy integration that Symfony provides for Doctrine[11-12]. 
Doctrine 2 provides natively support for multiple DB connections and object-relational mappings, we can safely use this tool within Symphony to handle read and writes to different databases without polluting the domain model of each of the services that take advantage of Doctrine. On another note, sharing the data model among different services helps as overcoming some situations where web services or messaging queues are not enough [11-12].

Finally, the probably most powerful concepts of Symphony are the Bundles. They are micro-applications inside the main application, being able to totally separate logics from different domains helps in keeping a clean separation of concerns and autonomously develops every single feature of the domain[11-12].

\section{Functional Aspects of SIMArech}

The actors of SIMArechcan be definedwithin four roles:

\section{The user or researcher:}

$\checkmark$ Dissemination and sharing of knowledge: Participates in increasing the visibility and the diffusion of the scientific knowledge; enriches the system by his/her scientific productions; makes it possible to quote the researchers with whom he/she was collaborating to realize a project...He/she follows and elaborates the activities of a research unit to which he/she belongs: team, laboratory / group, center.

$\checkmark$ Organization within the structure: the researcher must register in a research unit. $\mathrm{He} / \mathrm{she}$ can apply for membership in a laboratory / group or ask the administration to create a research structure if he/she has formed a group / laboratory, or change his/her membership so he/she will be part of a new group and only this group. "The researcher will be outside the group to which he/she belonged before."

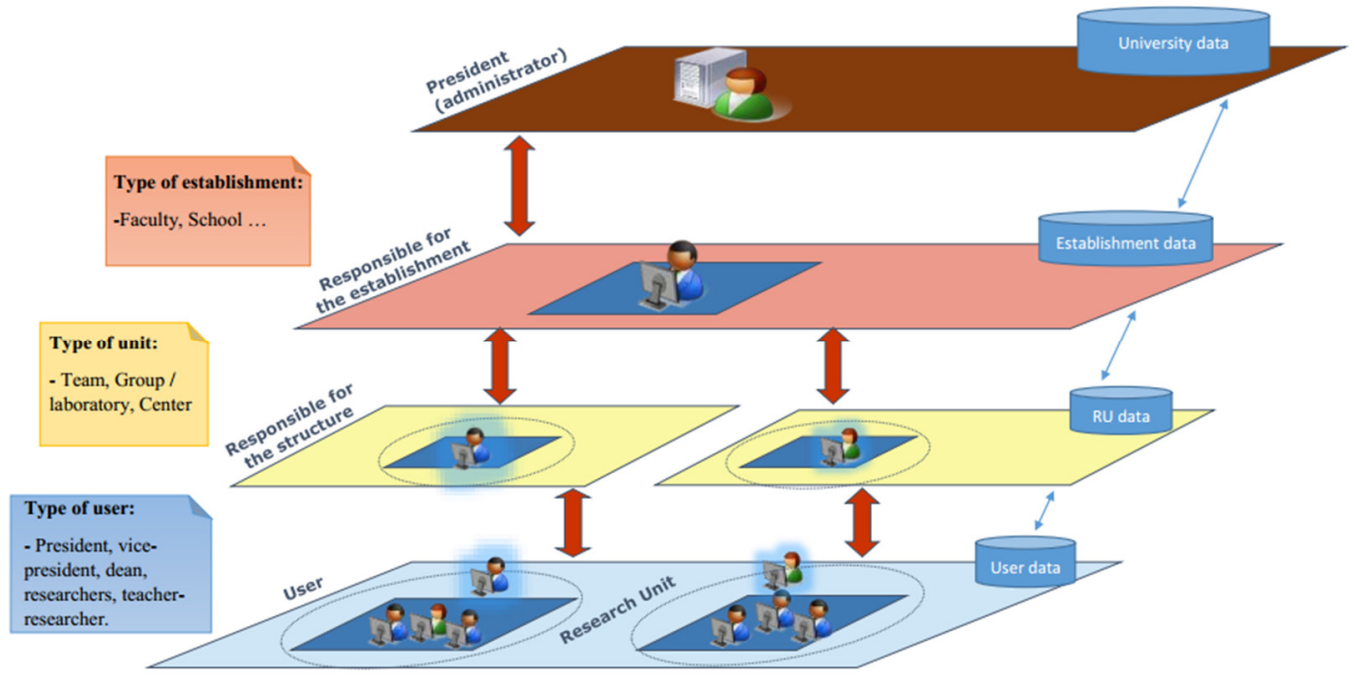

Figure 2: The actors of SIMArech 
The responsible of the research unit:

$\checkmark$ Dissemination and sharing of knowledge: participates in increasing the visibility and the diffusion of his/her own scientific knowledge, enriches the system by his/her scientific productions, makes it possible to quote the researchers with whom he/she was collaborating to realize a project ...

$\checkmark$ Team coaching: Records scientific publications, scientific events: congresses, symposiums, round tables, think tanks... for the account of the laboratory and / or research work. Proposes and defines current research work and set priorities: defines a clear search axis. Validates the necessary means (The need for scientific or computer equipment) to carry out a research activity, thus declares a fund offered by the major partner groups or ministries, associations for research, Moroccan fund, to evaluate the progress of his/her team's research.

$\checkmark$ Organization of Research Unit: the research unit can be transformed (URs and teams can explode, merge, regroup, etc ...)

\section{Responsible at the institution level:}

$\checkmark$ Dissemination and sharing of knowledge: participates in increasing the visibility and the diffusion of his/her own scientific knowledge, enriches the system by his/her scientific productions, makes it possible to quote the researchers with whom he/she was collaborating to realize a project ...

$\checkmark$ At the establishment level: Manages scientific productions of his/her research units, Evaluates the relevance of the adopted means to develop research within the research units. Develop the institution's budget.

\section{Responsible at the university level:}

The responsible at the university level plays the role of an internal animator. Manages all researchers belonging to the university and their scientific productions, controls, verifies, validates and accredits the information and operations...

\section{Modeling of the information system (input-output)}

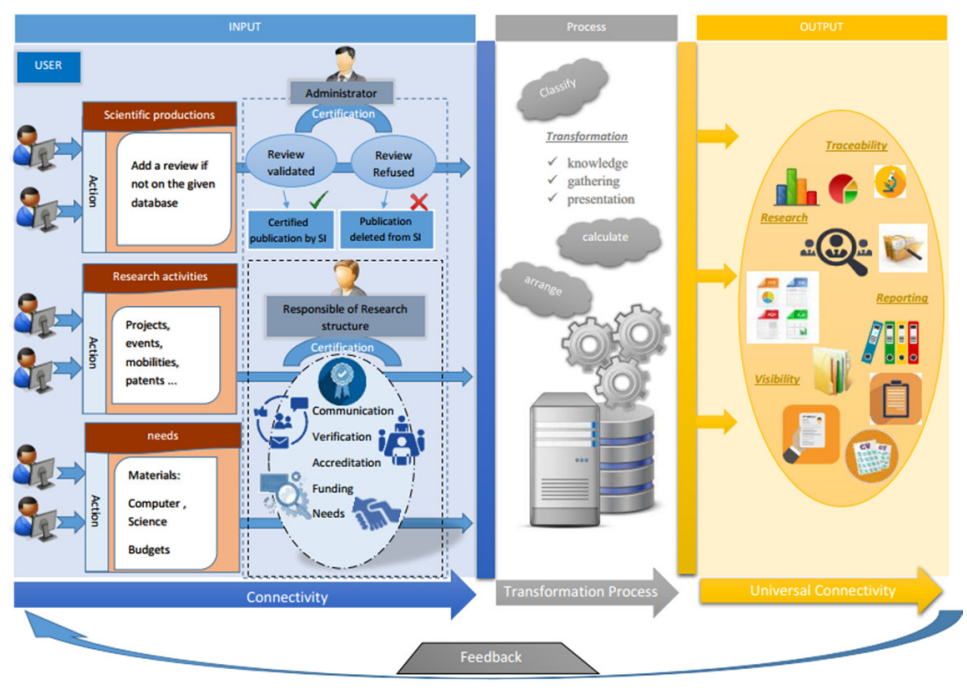

Figure 3 : input output of SIMArech 
The SIMArech information system includes inputs and outputs.

At the input level:

All the scientific activities (projects, manifestations, communications, movements, patents ...) entered by researchers, managers of structures, deans, and administrator.

$\checkmark$ The scientific activities entered by the researchers are verified by the responsible of structures.

$\checkmark$ The publications are verified and validated (according to the review) by the administrator.

\section{At the process level:}

Consists of a set of processing operations (classify, calculate, organize, etc.) of the data carried out by the SIMArech program.

\section{At the output level:}

$\checkmark \quad$ Visibility: CVs, scientific productions and follow-up of the national and international activities (missions, exchanges) the researchers, the research units and the university, as well as the distribution of research units and budgets.

$\checkmark$ Research: research in scientific productions of researchers.

$\checkmark$ Traceability: statistics, dashboards.

$\checkmark$ Reporting: CV, searchingfor structure accreditation file, Reports of scientific productions,

$\checkmark$ Decision: agreements, refusals, Conventions, accreditations, creation of research units,

$\checkmark$ Evaluation: The continuous analysis of scientific productions by those responsible for the structure makes it possible to declare their strengths and their weaknesses at the level of research units, as well as institutions, and universities in general. 


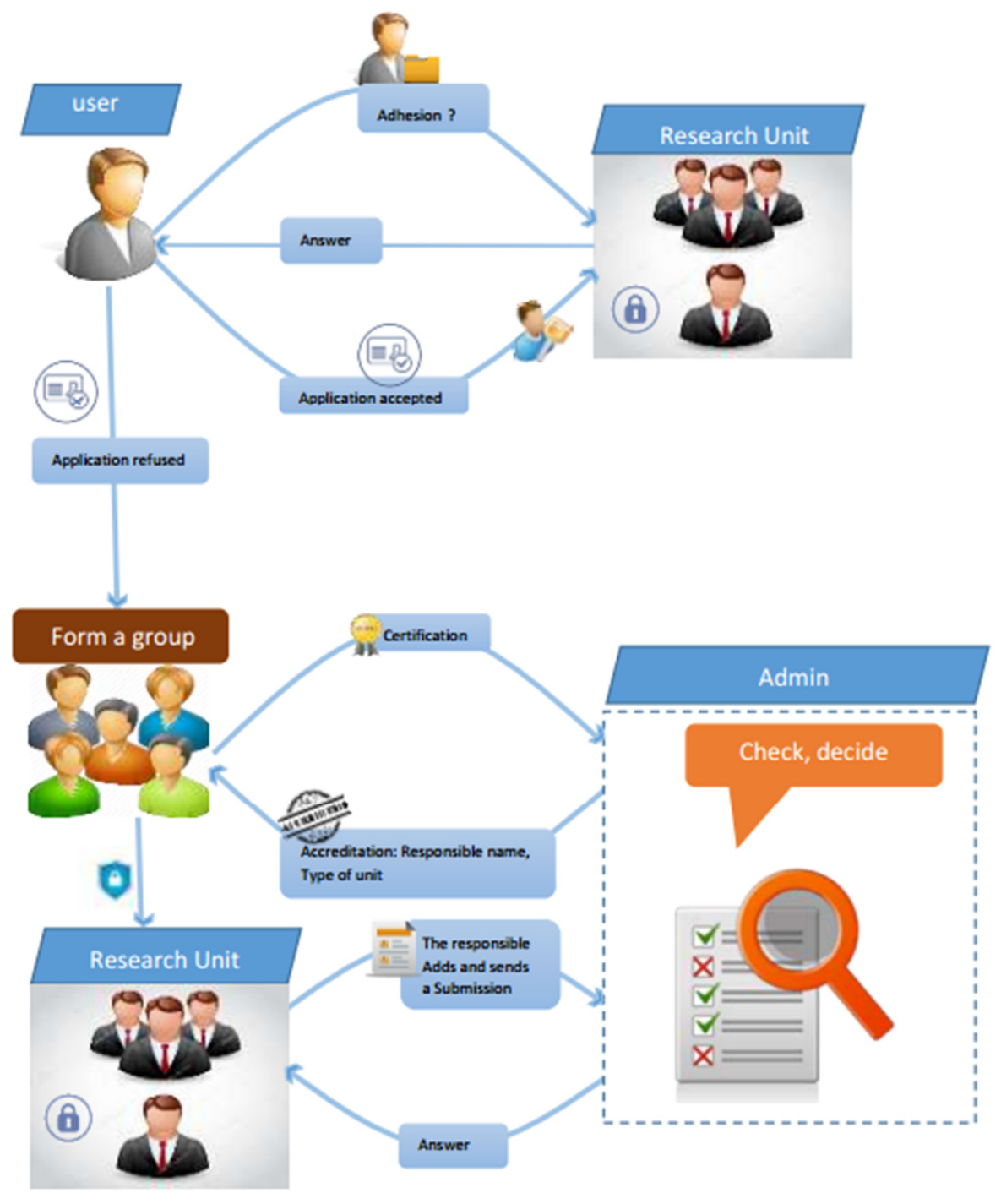

Figure 4: Management of the research unit by SIMArech

\section{Conclusion}

The scientific production of a university or a higher education institution is highly dynamic given that evolves on a daily basis. It is primordial for decision-makers to have a comprehensive dashboard that can provide them with timely information on research conducted within their institutions.

The SIMArech information system is an excellent collaborative tool that facilitates the research management in a digital way. With advanced menu-driven application tools for research performance assessment and monitoring of university research units, for journal indicators, ranking of universities and mapping, the information system have now reached a stage of high quality, reliable and very informative instruments in research evaluation practice.

\section{REFERENCES}

[1] Waltman, L., van Eck, N.J., van Leeuwen, T.N., Visser, M.S. and van Raan, A.F.J. (2011) Towards a new crown indicator: an empirical analysis. Scientometrics 87, 467-481 
[2] VanRaan, A.F.J. (2004) Measuring science. Capita selecta of current main issues. In: Handbook of Quantitative Science and Technology Research (Moed, H.F., Glänzel, W. and Schmoch, U., eds), pp. 1950, Kluwer Publishers, Dordrecht

[3] Butler, L. and Visser, M.S. (2006) Extending citation analysis to non-source items. Scientometrics 66, $327-343$

[4] vanRaan, A.F.J. (2006) Performance-related differences of bibliometric statistical properties of research groups: cumulative advantages and hierarchically layered networks. Journal of the American Society for Information Science and Technology 57, 1919-1935

[5] CABRERA J, DE LA SOTA J, GONZÁLEZ A, GUERRERO H, PRESMANES B, "El papel de los expertos enciencia y tecnología", Revista Madri+d. Monografía 5, p 32-57, 2002

[6] MOED H, Bibliometric indicators reflect publication and management strategies, Scientometrics, V 47, $\mathrm{N}$ 2, p. 323-346, 2000

[7] WARTA K, CHARLET V, Enquête 2002 auprès des laboratoires angevins, pour l'Observatoire de la RechercheAngevine, rapport Technopolis France, 2003

[8] ZITT M, BAUIN S, FILLIATREAU G, Production coopérative d'indicateurs inter-institutionnels de politiquescientifique. Indicateurs bibliométriques des institutions publiques de recherche, rapport OST,2002

[9] Natalya Prokofyevaa, Victoria BoltunovaaAnalysis and Practical Application of PHP Frameworks inDevelopment of Web Information SystemsProcedia Computer Science 10451-56 (2017)

[10] 10. SensioLabs. The Symfony Book v. 3.1; 2016. p. 4-68. Available: http://symfony.com/pdf/Symfony_book_3.1.pdf.

[11] 11. PHP Documentation. PHP's memory_get_included_files. Available: http://php.net/manual/en/function.get-included-files.php.

[12] PHP Documentation. PHP's memory_get_usage function. Available: http://php.net/manual/en/function.memory-get-usage.php. 